O. Diall ${ }^{1}$

Z. Bocoum ${ }^{1}$

B. Diarra ${ }^{1}$

Y. Sanogo ${ }^{1}$

Z. Coulibaly ${ }^{1}$

Y. Waïgalo ${ }^{2}$

\title{
Épidémiologie de la trypanosomose à T. evansi chez le dromadaire au Mali : résultats d'enquêtes parasitologiques et cliniques
}

DIALL (O.), BOCOUM (Z.), DIARRA (B.), SANOGO (Y.), COULIBALY (Z.), WAIGALO (Y.). Épidémiologie de la trypanosomose à $T$. evansi chez le dromadaire au Mali : résultats d'enquêtes parasitologiques et cliniques. Revue Élev. Méd. vét. Pays trop., 1993, 46 (3) : 455-461

L'épidémiologie de la trypanosomose du dromadaire a été étudiée au Mali dans les régions d'élevage. Basée sur des enquêtes parasitologiques et cliniques, elle a révélé des taux d'infection globaux de $\mathbf{9 , 5}$ p.100 (29/305) dans le Sahel occidental (zone I) et de 4,5 p.100 (28/627) dans les zones de Tombouctou et de Gao (zone II). La proportion de troupeaux contaminés a été de 55 p.100 en région I et de 68 p.100 en région II. Certains troupeaux se trouvent infectés à plus de 50 p.100 de leurs effectifs. Nos enquêtes ont montré une tendance à l'accroissement de la prévalence parasitologique avec l'âge. Alors que celle-ci est virtuellement nulle chez les jeunes de moins d'un an, elle s'accroît avec l'âge pour atteindre son maximum dans la classe de 2-5 ans. Les auteurs montrent que l'infection a un effet significativement négatif sur l'hématocrite et sur l'état général, confirmant la pathogénicité de Trypanosoma evansi pour le dromadaire. Ce trypanosome a été le seul (ou presque le seul) rencontré chez le dromadaire au Mali. Il ne semble pas causer d'infections patentes chez d'autres espèces animales, évoluant dans le même milieu.

Mots-clés : Dromadaire - Trypanosomose - Trypanosoma evansi - Épidémiologie - Enquête pathologique - Mali - Sahel.

\section{INTRODUCTION}

Le cheptel camelin malien était estimé à 230000 têtes en 1988 (5). II évolue dans une zone entièrement située au nord du $14 \mathrm{e}$ parallèle, hors d'atteinte des tsé-tsé. C'est donc principalement la trypanosomose à $T$. evansi, mécaniquement transmise par des insectes piqueurs, qui affecte le dromadaire.

A la fin du 19 e siècle, CAZALBOU (3) décrivit à Tombouctou une maladie du dromadaire, dont l'identité avec le "surra" observé ailleurs en Asie et en Afrique fut ultérieurement établie. II ressort des rapports du service de l'Elevage et des Industries animales du Soudan français (12) que la trypanosomose à $T$. evansi, localement appelée "M'bori", constituait la pathologie cameline dominante et ceci à un point tel que la plupart des autres affections dont pouvait souffrir cet animal étaient considérées comme des maladies de sortie ou des séquelles du "M'bori". Quant aux éleveurs, ils font parfaitement la rela-

\footnotetext{
1. Laboratoire central vétérinaire de Bamako, B.P. 2295, Mali.

2. Direction régionale de l'Elevage de Gao, Mali.

Reçu le 10.9.1992, accepté le 14.9.1993.
}

tion entre son apparition et la présence de mouches piqueuses, notamment le long du fleuve Niger et autour des mares et des lacs.

II a fallu cependant attendre 1985 pour qu'un programme d'enquêtes sur la prévalence de cette affection puisse être réalisé au Mali jusqu'en 1991.

Mais compte tenu de l'étendue de l'aire d'élevage du dromadaire et de l'insécurité qui y régnait ces dernières années, des sites importants comme Kidal et Menaka n'ont pu être visités et d'autres n'ont pu l'être qu'une seule fois.

Au total, nos enquêtes ont permis l'examen de 1560 échantillons sanguins. Dans leur conception, elles prévoient la possibilité de déterminer le taux d'infection des animaux, les facteurs de variation de celui-ci, ainsi que la relation entre l'infection et certains paramètres cliniques (anémie, état général).

\section{Aire de prospection - Environnement}

Elle est répartie en deux régions ou zones distinctes le nord-ouest ou Sahel occidental (région l), le nord et le nord-est correspondant aux zones de Tombouctou et de Gao (région II). Chacune de ces régions est à son tour divisée en secteurs, qui ne coïncident pas toujours avec les traditionnels secteurs d'élevage. Deux sites visités en Mauritanie ont été rattachés par commodité au secteur frontalier de Nara au Mali (carte 1).

La zone I se situe entre les isohyètes 400 et $600 \mathrm{~mm}$. Le fonds végétal y est représenté par un tapis herbacé plus ou moins abondant et un couvert arbustif essentiellement constitué d'Acacia spp. Les troupeaux camelins transhumants des deux "Hodhs" mauritaniens y trouvent leurs pâturages de saison sèche. Leurs mouvements sont dictés par le régime des pluies et orientés du nord au sud en fin de saison sèche froide (décembre-janvier) et du sud au nord, en début de saison pluvieuse (juillet). Ils passent donc 6-8 mois au Mali à l'intérieur de la zone d'étude et 4-6 mois en Mauritanie au nord de celle-ci. Ce dernier séjour permet aux troupeaux transhumants de limiter les contacts avec les insectes piqueurs qui abondent en cette période, notamment autour des nombreuses mares alimentées par les pluies. 


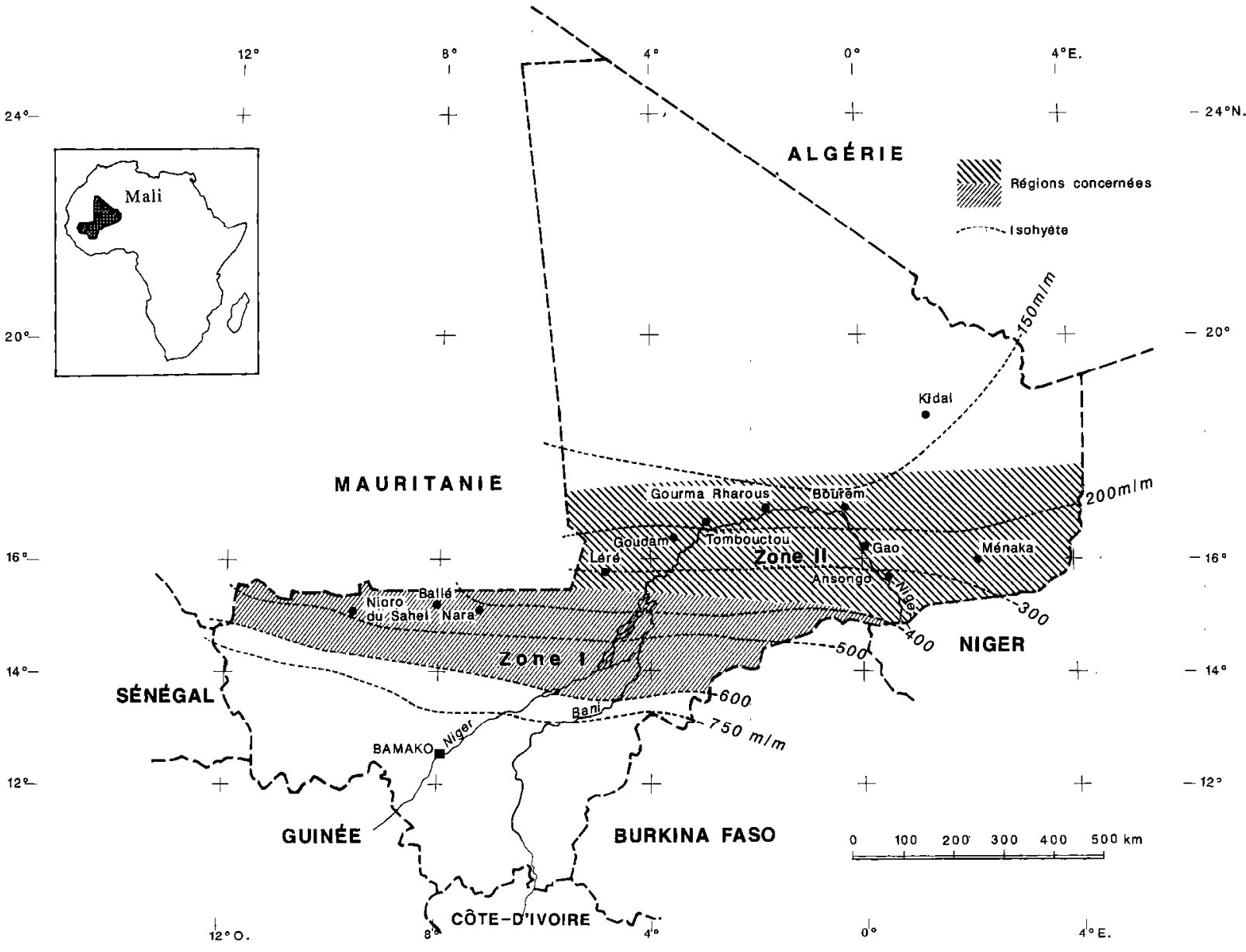

Carte 1 : Zones d'enquête sur la trypanosomose à $\mathrm{T}$. evansi chez le dromadaire au Mali.

La zone II englobe les traditionnelles zones d'élevage du dromadaire (Tombouctou et Gao) et se situe entre les isohyètes $200-400 \mathrm{~mm}$, certains sites, comme dit plus haut, étant exclus (isohyète 150).

En saison sèche, les camelins exploitent les pâturages herbacés et arbustifs dans la vallée du Niger et autour des mares et des lacs tributaires du même fleuve.

En saison pluvieuse, ils remontent plus au nord, mais restent généralement dans les limites du territoire malien.

\section{MATÉRIEL ET MÉTHODES}

\section{Matériel animal et méthodologie des enquêtes}

Le matériel animal est composé de dromadaires d'élevage transhumant ou de transport. Dans la région I, tous les troupeaux rencontrés, à l'exception de deux à Nioro du Sahel (Mali), sont d'origine mauritanienne alors que les chameaux de transport appartiennent pour l'essentiel à des Maliens. En région II, en revanche, tous les effectifs camelins enquêtés sont d'origine malienne.
En marge de ces enquêtes, la recherche de trypanosomes a été effectuée sur un nombre restreint de bovins, petits ruminants, équins et asins.

Pour chaque animal, il a été enregistré : l'âge, le sexe, l'état d'embonpoint, la saison à laquelle s'est déroulée l'enquête et quelques données sur le troupeau et son statut (propriétaire, origine, lieu de rencontre, itinéraire). Le tableau I résume les 1560 enregistrements effectués.

\section{Prélèvements}

Le choix des troupeaux pour les prélèvements relève à la fois du hasard des rencontres et de la compréhension des éleveurs maures (région I) et touareg (région II). Les nomades sont généralement opposés à la saignée de leurs animaux. Cependant, la session gratuite de produits vétérinaires et de médicaments à usage humain a souvent permis d'obtenir leur collaboration. Pour les chameaux de transport rencontrés au niveau des foires, l'obtention d'échantillons est beaucoup plus difficile et l'intervention des autorités locales a été souvent nécessaire pour obtenir, des différents propriétaires, un minimum de coopération. 
TABLEAU I Répartition géographique des 1560 camelins examinés.

\begin{tabular}{|c|c|c|c|c|c|c|c|c|c|c|c|c|}
\hline & Nara & Balle & Nioro & $\begin{array}{c}\text { Total } \\
\text { région I }\end{array}$ & Lere & Goundam & Tombouctou & Rharous & Bourem & Gao & Ansongo & $\begin{array}{c}\text { Total } \\
\text { région II }\end{array}$ \\
\hline $\begin{array}{c}\text { Nara } \\
\text { Ballé } \\
\text { Nioro } \\
\text { Total } \\
\text { région I }\end{array}$ & 562 & 105 & 100 & 767 & & & & & & 1 & & \\
\hline $\begin{array}{c}\text { Léré } \\
\text { Goundam } \\
\text { Tombouctou } \\
\text { Rharous } \\
\text { Bourem } \\
\text { Gao } \\
\text { Ansongo } \\
\text { Total } \\
\text { région II }\end{array}$ & & & & & 115 & 102 & 29 & 159 & 39 & $\begin{array}{c}219 \\
1\end{array}$ & 130 & 793 \\
\hline $\begin{array}{c}0-1 \text { an } \\
1-2 \text { ans } \\
2-5 \text { ans } \\
5 \text { ans } \\
\text { et plus }\end{array}$ & $\begin{array}{r}13 \\
19 \\
146 \\
384\end{array}$ & $\begin{array}{l}19 \\
19 \\
19 \\
48\end{array}$ & $\begin{array}{r}20 \\
7 \\
11 \\
62\end{array}$ & $\begin{array}{r}52 \\
45 \\
176 \\
494\end{array}$ & $\begin{array}{r}8 \\
16 \\
34 \\
57\end{array}$ & $\begin{array}{r}0 \\
4 \\
35 \\
63\end{array}$ & $\begin{array}{r}0 \\
1 \\
1 \\
27\end{array}$ & $\begin{array}{l}10 \\
17 \\
48 \\
84\end{array}$ & $\begin{array}{r}2 \\
8 \\
8 \\
21\end{array}$ & $\begin{array}{r}1 \\
22 \\
77 \\
119\end{array}$ & $\begin{array}{r}1 \\
28 \\
17 \\
84\end{array}$ & $\begin{array}{r}22 \\
96 \\
220 \\
455\end{array}$ \\
\hline $\begin{array}{c}\text { Mâles } \\
\text { Femelles }\end{array}$ & $\begin{array}{l}441 \\
121\end{array}$ & $\begin{array}{l}34 \\
71\end{array}$ & $\begin{array}{l}38 \\
62\end{array}$ & $\begin{array}{l}513 \\
254\end{array}$ & $\begin{array}{l}43 \\
72\end{array}$ & $\begin{array}{l}59 \\
43\end{array}$ & $\begin{array}{r}21 \\
8\end{array}$ & $\begin{array}{l}85 \\
74\end{array}$ & $\begin{array}{l}14 \\
25\end{array}$ & $\begin{array}{r}135 \\
84\end{array}$ & $\begin{array}{l}41 \\
89\end{array}$ & $\begin{array}{l}398 \\
395\end{array}$ \\
\hline
\end{tabular}

\section{Echantillonnage}

II a été effectué sans aucune discrimination. Les troupeaux présentés étaient saignés dans la quasi-totalité de leurs effectifs, à l'exception de certains mâles dont la contention était difficile. Tous les chameaux de transport auxquels nous avons eu accès ont été également saignés. Environ $10 \mathrm{ml}$ de sang sont prélevés dans un tube sans anticoagulant, puis $1 \mathrm{ml}$ de ce sang est immédiatement transféré dans un deuxième tube hépariné. Le premier tube sert à la récolte de sérum, le second à la recherche de trypanosomes.

\section{Méthodologie des examens sanguins - Technique de diagnostic}

A partir de chaque échantillon, un frottis et une goutte épaisse ont été préparés et un tube capillaire rempli de sang en vue de la centrifugation.

La recherche de trypanosomes en "frais" est effectuée en utilisant la technique de centrifugation hématocrite (11) ; les examens microscopiques ont été généralement réalisés au fur et à mesure des prélèvements grâce à l'utilisation sur place d'un groupe électrogène. Ils sont précédés par la lecture des valeurs de l'hématocrite.

\section{Examens positifs}

Le degré d'infection est estimé selon l'échelle logarithmique proposée par MURRAY et al. (11) mais résumée ici en quatre croix :

Une $+=1-10$ trypanosomes par lame ;

Deux $+=1$ trypanosome dans 10 champs à 1 trypanosome par champ ;

Trois $+=1-10$ trypanosomes par champ ;

Quatre $+=$ plus de 10 trypanosomes par champ.

Les résultats globaux sont reportés aux tableaux I et II.

Les frottis et les gouttes épaisses ont été fixés et colorés par les méthodes usuelles (10).

La parasitémie chez un sujet est considérée comme forte, lorsque chaque champ microscopique compte au moins un trypanosome.

Un troupeau est dit contaminé, lorsqu'il héberge au moins un animal trouvé porteur d'au moins un trypanosome.

Au cours des enquêtes effectuées en 1990-91, l'épreuve d'inoculation à la souris (8) a été utilisée. Mais compte tenu du nombre limité de souris dont on pouvait disposer sur le terrain, seuls les sujets franchement anémiés 
(hématocrite< 20 p.100), ont été soumis à cette épreuve. La recherche de trypanosomes a été quotidiennement effectuée chez les souris, pendant 30 jours à partir du troisième jour après l'inoculation.

\section{Analyse des données}

Les taux d'infection ont été calculés et leurs facteurs de variation (région, secteur, saison, âge) étudiés en leur appliquant les tests de comparaison de deux ou de plusieurs probabilités. Ces mêmes tests ont été utilisés pour déterminer l'effet des infections à $T$. evansi sur l'hématocrite et l'état général. L'étude de la variation du taux d'infection selon la région, le secteur et l'âge a été effectuée sur la base des données recueillies au cours de l'enquête réalisée en 1990-91, au cours de laquelle tous les secteurs ont été couverts en 3 mois. En revanche, l'étude de la variation saisonnière n'a été possible que pour le secteur de Nara, le seul ayant fait l'objet de plusieurs visites s'étalant de 1985 à 1990 .

\section{RÉSULTATS}

\section{Variation du taux d'infection global}

En région I, sur les 305 camelins soumis à l'enquête en 1990-91, 29 se sont avérés porteurs de trypanosomes $(9,5$ p.100) et 5 troupeaux sur 8 contaminés.

En région II, sur 627 sujets enquêtés, 28 sont positifs $(4,5$ p. 100) et 12 troupeaux sur 17 contaminés.

La région I s'est avérée significativement plus infectée que la région II $\left(\chi^{2}=8,22 ; p<0,1 p .100\right)$. Le tableau II montre que dans chacune des régions, on trouve des secteurs très infectés et d'autres qui le sont beaucoup moins. C'est ainsi qu'en région I, le secteur de Nara apparaît fortement infecté avec un taux de 25 p.100 tandis que ceux de Ballé et Nioro, avec des taux de 0,9 p. 100 et 3 p. 100 respectivement, le sont beaucoup moins. En région II, les taux les plus élevés sont enregistrés dans les secteurs d'Ansongo (7,7 p.100), Gao (7,5 p.100) et Léré (5,2 p.100).

\section{Variation du taux d'infection en fonction de la saison}

Le tableau III montre les taux d'infection enregistrés dans le secteur de Nara pour différentes saisons et années.

En 1985, le taux d'infection s'est montré significativement plus élevé en saison pluvieuse qu'en fin de saison sèche $\left(\chi^{2}=13,57 ; p<0,1\right.$ p.100).

Paradoxalement, en saison pluvieuse de 1987, on n'a pas pu déceler le moindre cas d'infection sur un échantillon de 78 animaux de transport. Quant aux autres
TABLEAU II Taux d'infection des animaux par région et par secteur.

\begin{tabular}{|c|c|c|c|c|}
\hline Région & Secteur & $\begin{array}{c}\text { Animaux } \\
\text { examinés }\end{array}$ & $\begin{array}{c}\text { Examens } \\
\text { positifs }\end{array}$ & $\begin{array}{c}\text { Pourcentage } \\
\text { de positifs }\end{array}$ \\
\hline & Nara & 100 & 25 & 25 \\
I & Ballé & 105 & 1 & 0,9 \\
& Nioro & 100 & 3 & 3 \\
& Total I & 305 & 29 & 9,5 \\
\hline II & Léré & 115 & 6 & 5,2 \\
II & Goundam & 102 & 4 & 3,9 \\
II & Tombouctou & 29 & 0 & 0 \\
II & Rharous & 159 & 4 & 2,5 \\
II & Bourem & 39 & 0 & 0 \\
II & Gao & 53 & 4 & 7,5 \\
II & Ansongo & 130 & 10 & 7,7 \\
& Total II & 627 & 28 & 4,5 \\
\hline
\end{tabular}

TABLEAU III Taux d'infection dans le secteur de Nara selon l'année et la saison.

\begin{tabular}{|c|c|c|c|c|}
\hline Année & Saison & $\begin{array}{c}\text { Animaux } \\
\text { examinés }\end{array}$ & $\begin{array}{c}\text { Examens } \\
\text { positifs }\end{array}$ & $\begin{array}{c}\text { Pourcentage } \\
\text { de positifs }\end{array}$ \\
\hline 1985 & 3 & 100 & 3 & 3 \\
& 4 & 95 & 20 & 21 \\
1987 & 2 & 135 & 18 & 13 \\
& 4 & 78 & 0 & 0 \\
1988 & 4 & 54 & 6 & 11 \\
1990 & 1 & 100 & 25 & 25 \\
\hline
\end{tabular}

1. Début de saison sèche (octobre-décembre) ; 2. Milieu de saison sèche (janvier-mars); 3. Fin de saison sèche (avril-juin) ; 4. Saison pluvieuse (juillet-septembre).

années (1988 et 1991), elles n'ont connu qu'une seule visite chacune, ce qui ne permet pas de comparaison statistique entre saisons. Cependant, sur l'ensemble de nos visites, les prévalences les plus élevées ont été enregistrées en saison pluvieuse de 1985 (21 p.100) et en début de saison sèche 1990 (25 p.100).

\section{Variation du taux d'infection en fonction de l'âge}

Les effectifs enquêtés en 1990-1991, partagés en 4 classes d'âge, ont donné les taux d'infection suivants :
0-1 an
0,0 p.100 (0/61)
1-2 ans
5,2 p.100 (6/115)
2-5 ans
8,0 p.100 (17/211)
5 ans et plus
6,2 p.100 (34/545) 
Le taux d'infection s'est avéré nul chez les chamelons de moins d'un an. II a connu une augmentation dans la classe d'âge de 2-5 ans, puis une baisse dans la classe de 5 ans et plus. La comparaison des pourcentages n'a pas révélé de différence statistiquement significative.

\section{Trypanosomes rencontrés et degré d'infection}

Tous les trypanosomes de type brucei isolés dans le cadre du présent travail sont de "forme longue". De ce fait, et compte tenu de la localisation géographique, ils ont été considérés comme appartenant à l'espèce $T$. evansi.

Sur les 109 cas de trypanosomose rencontrés chez le dromadaire pour l'ensemble des enquêtes, 108 sont dus à $T$. evansi en infection pure et un seul cas dû à une infection mixte $T$. evansi/T. vivax.

L'utilisation de la méthode de centrifugation hématocrite (HCT) a permis d'apprécier le degré d'infection à travers un comptage des trypanosomes par lame ou par champ microscopique. Ainsi, il s'est avéré que 41 p.100 des sujets infectés présentent une forte parasitémie.

\section{Infections à T. evansi chez d'autres espèces animales}

Au cours des enquêtes effectuées dans le secteur de Nara, la recherche de trypanosomes a été réalisée chez un nombre restreint d'asins (49), de bovins (26), de petits ruminants (6) et d'équins (3). Aucun cas d'infection à $T$. evansi n'a pu être décelé.

\section{Effet clinique de la trypanosomose à $T$. evansi}

Les effets de l'infection à $T$. evansi ont été étudiés sur l'hématocrite et l'état général des animaux.

\section{Anémie et hématocrite}

Pour mesurer l'effet de l'infection sur l'hématocrite, il a été procédé à la comparaison des moyennes entre sujets infectés et non infectés à l'intérieur d'un même troupeau d'une part, et entre troupeaux contaminés et indemnes d'autre part. Les résultats exprimés dans les tableaux IV et $V$ montrent une différence significative avec une probabilité inférieure ou égale à 0,1 p.100 dans le premier cas et inférieure à 5 p.100 dans le second.

Dans cette étude, nous avons considéré comme anémiés tous les animaux ayant un hématocrite inférieur à 23 p.100. Car la moyenne de l'hématocrite chez les animaux non porteurs de trypanosomes dans les différents secteurs a varié entre 22,7 p.100 et 26,9 p.100 au cours de l'enquête 1990-1991.

Ainsi, sur la base des données recueillies au cours de l'enquête 1990-1991, la comparaison entre le pourcentage d'animaux infectés et anémiés d'une part, et le pourcentage d'animaux anémiés et négatifs d'autre part, a donné les résultats suivants :

- 87,5 p.100 (49/56) des animaux infectés sont anémiés ;

- 25,7 p.100 (225/876) des animaux négatifs sont anémiés $(\varepsilon=9,8$; différence significative à moins de 0,1 p.100).

TABLEAU IV Données hématologiques en rapport avec la présence de T. evansi (secteur de Nara).

\begin{tabular}{|c|c|c|c|c|c|}
\hline \multicolumn{1}{|c|}{ Date } & $\begin{array}{c}\text { Statut } \\
\text { parasitologique }\end{array}$ & $\begin{array}{c}\text { Nombre } \\
\text { sujets }\end{array}$ & Amplitude & $\begin{array}{c}\text { Hématocrite } \\
\text { moyen }\end{array}$ & $t$ \\
\hline Septembre 1985 & Nég./Pos. & $86 / 15$ & $10-36 / 11-21$ & $21,3 / 17,4$ & 3,53 \\
Mars 1987 & Nég./Pos. & $117 / 18$ & $17-36 / 17-30$ & $27,2 / 24,0$ & 3,35 \\
Décembre 1990 & Nég./Pos. & $75 / 25$ & $18-31 / 15-27$ & $23,9 / 20,0$ & 5,45 \\
\hline
\end{tabular}

TABLEAUV Moyennes de l'hématocrite chez des troupeaux positifs et négatifs (secteur de Nara).

\begin{tabular}{|c|c|c|c|c|c|}
\hline \multirow{2}{*}{ Date } & \multirow{2}{*}{$\begin{array}{l}\text { Numéro } \\
\text { troupeau }\end{array}$} & \multirow{2}{*}{$\begin{array}{c}\text { Statut } \\
\text { parasitologique }\end{array}$} & \multirow{2}{*}{ Effectif } & \multicolumn{2}{|c|}{ Hématocrite } \\
\hline & & & & Amplitude & Moyenne \\
\hline $\begin{array}{l}\text { Mars } 1987 \\
\text { Mars } 1987 \\
\text { Mars } 1987 \\
\text { Mars } 1987\end{array}$ & $\begin{array}{l}1 \\
2 \\
3 \\
4\end{array}$ & $\begin{array}{c}\text { Positif }(21,6 \%) \\
\text { Positif }(59,0 \%) \\
\text { Négatif } \\
\text { Négatif }\end{array}$ & $\begin{array}{l}37 \\
17 \\
30 \\
23\end{array}$ & $\begin{array}{l}17-33 \\
19-30 \\
23-34 \\
24-36\end{array}$ & $\begin{array}{l}24,9 \pm 0,7 \\
25,0 \pm 0,7 \\
27,6 \pm 0,5 \\
29,7 \pm 0,7\end{array}$ \\
\hline
\end{tabular}




\section{O. Diall Z. Bocoum B. Diarra Y. Sanogo Z. Coulibaly Y. Waïgalo}

A partir de ce taux, si l'on admet que 26 p.100 des animaux infectés font une anémie d'origine non trypanosomienne, on peut considérer qu'au moins 61 p.100 des animaux infectés présentent une anémie attribuable à $T$. evansi.

\section{Influence des infections à $T$. evansi sur l'état général}

L'état général a été défini sur la base de l'observation des animaux et de l'avis des propriétaires. Ainsi, 64 p.100 (36/56) des animaux infectés présentent un mauvais été général contre 39 p.100 (343/876) des animaux parasitologiquement négatifs $(\varepsilon=3,69$; différence significative à moins de $0,1 \mathrm{p} .100$ ). On peut donc estimer qu'au moins 25 p.100 des animaux infectés ont un mauvais état général dû à la trypanosomose à $T$. evansi.

\section{DISCUSSION ET CONCLUSION}

Les enquêtes réalisées en 1990-1991 ont mis en évidence des pourcentages moyens d'animaux positifs de 9,5 p.100 pour la région I et de 4,5 p.100 seulement pour la région II.

La trypanosomose cameline apparaît donc plus fréquente dans le Sahel occidental, où les précipitations annuelles varient entre 400 et $600 \mathrm{~mm}$, que dans le nord et le nordest où elles sont en moyenne inférieures à $400 \mathrm{~mm}$.

D'une manière générale, nos résultats montrent la présence de $T$. evansi dans tous les secteurs de l'aire d'étude. Les nombreuses prospections effectuées à Nara en région I signalent une forte endémicité à $T$. evansi dans ce secteur.

Les secteurs de Bourem et de Tombouctou qui paraissent négatifs sont ceux dans lesquels le nombre de prélèvements est le plus faible et où un seul troupeau était concerné.

Le pourcentage d'animaux positifs s'est avéré très variable d'un troupeau à l'autre. Au cours d'une même prospection, on a pu rencontrer des troupeaux contaminés à plus de 50 p.100 et d'autres apparemment indemnes (tableau $\mathrm{V}$ ). Ce phénomène est certainement lié au caractère mécanique de la transmission de l'infection.

L'analyse de l'influence de la saison sur le taux d'infection appliquée au secteur de Nara montre des taux significativement plus élevés en début de saison sèche (25 p.100) et en saison pluvieuse (21 p.100).

A ce propos, il convient de préciser que les enquêtes de saison pluvieuse, en zone I, concernent exclusivement les chameaux de transport, qui bénéficient généralement de traitements trypanocides individuels, notamment à l'orée de cette saison. En revanche, les enquêtes de sai- son sèche s'adressent aussi et surtout aux chameaux d'élevage transhumant qui remontent en Mauritanie dès la mi-juillet.

II est généralement connu que le pic des infections à $T$. evansi se situe en saison des pluies et à la fin de celle-ci, à l'époque où les insectes piqueurs (taons et stomoxes) sont particulièrement abondants $(2,9)$.

Bien qu'elle n'ait pas révélé de différence statistiquement significative entre les classes d'âge, l'étude a montré une tendance du taux d'infection à s'accroître avec l'âge atteignant un maximum dans la classe de 2-5 ans. Les occasions de s'infecter croissant avec l'âge, les chameaux adultes ( 5 ans et plus) bénéficient probablement d'une résistance acquise à la faveur des infections répétées, subies, ce qui expliquerait la tendance à la baisse dans ce dernier groupe.

L'absence virtuelle de l'infection chez les chamelons de moins d'un an confirme les résultats obtenus en Mauritanie par l'équipe du Dr JACQUIET (4). Ceci pourrait résulter d'une résistance naturelle. En tout cas, il est peu probable que ceci soit le résultat d'une immunité acquise à travers le colostrum, car un seul chamelon sur les 61 examinés a donné une sérologie positive à la trypanosomose par la réaction d'agglutination rapide, CATT/T. evansi (DIALL, inédit).

Trypanosoma evansi a été presque le seul trypanosome rencontré au cours de nos enquêtes chez le dromadaire au Mali et ne semble pas causer d'infections patentes chez les autres espèces animales évoluant dans le même milieu.

En Mauritanie également, la présence de Trypanosoma evansi chez des espèces autres que le dromadaire n'a pu être établie à travers les nombreux" examens effectués, notamment chez les bovins et les petits ruminants (4).

La recherche de Trypanosoma evansi chez les petits ruminants s'est avérée aussi infructueuse au Soudan, malgré la détection dans leurs sérums d'anticorps spécifiques (2).

Enfin, des moutons et des chèvres artificiellement infectés avec des souches de Trypanosoma evansi isolées sur dromadaire développent généralement de faibles parasitémies $(2,4)$.

Ces faits expérimentaux font penser au rôle potentiel des petits ruminants dans la transmission de Trypanosoma evansi. Mais celui-ci ne pourra être confirmé sans la mise en évidence du parasite. Des enquêtes impliquant des effectifs plus importants sont donc nécessaires.

Chez les camelins infectés et non traités, anémie et baisse de condition sont des signes quasi constants (6) qui ont été utilisés pour apprécier l'effet pathogène du parasite dans les secteurs visités. L'estimation de la valeur normale de l'hématocrite chez le dromadaire montre que 
celle-ci varie entre 20 et 33 p.100 avec une moyenne de 27 p.100 (1).

Les valeurs enregistrées au cours de ces enquêtes sont généralement inférieures à cette moyenne, même pour des sujets apparemment non infectés. La comparaison entre sujets positifs et sujets négatifs a permis de mettre en évidence l'effet significativement négatif de l'infection sur l'hématocrite et l'état général.

Les résultats obtenus donnent une vue d'ensemble sur l'incidence de la trypanosomose cameline au Mali. Les faibles pourcentages observés dans les zones traditionnelles d'élevage du dromadaire (Gao, Tombouctou) pourraient s'expliquer par leur position très septentrionale et l'assèchement de nombreux lacs et mares, consécutivement aux récentes et nombreuses années de sécheresse.

La réalisation en cours de projets de réhabilitation de certains de ces points d'eau pourrait modifier cette tendance. Dès que les conditions le permettront, cette étude devra donc être complétée par une enquête sur les insectes piqueurs de ces régions.

\section{REMERCIEMENTS}

Notre étude a été réalisée grâce à l'assistance financière de la CEE dans le cadre du projet TS-0071 et de I'USAID dans le cadre du projet sectoriel de l'Elevage. Sur le terrain, son exécution n'aurait pu être possible sans la collaboration du personnel de l'Elevage en poste dans les différents secteurs visités ; qu'il veuille bien accepter l'expression de notre profonde gratitude. Nous voudrions également remercier le Dr Boubacar M'baye SECK, directeur du Laboratoire central vétérinaire, pour l'intérêt qu'il n'a cessé de porter à cette étude.

DIALL (O.), BOCOUM (Z.), DIARRA (B.), SANOGO (Y.), COULIBALY (Z.), WAIGALO (Y.). Epidemiology of trypanosomosis due to Trypanosoma evansi in dromedary camel in Mali: Results of parasitological and clinical surveys. Revue Elev. Méd. vét. Pays trop., 1993, 46 (3) : $455-461$

\begin{abstract}
An epidemiological study of trypanosomosis was conducted in the rearing areas of dromedary camels in Mali. According to the parasitological and clinical surveys performed, the overall infection rates were 9.5 $\%(29 / 305)$ in Western Sahel (region $I)$ and $4.5 \%(28 / 627)$ in the areas of Tombouctou and Gao (region II). The proportion of contaminated herds was $55 \%$ in region I and $68 \%$ in region II and in some herds the infection rate exceeded $50 \%$. The surveys showed a trend for increasing parasitological prevalence with age. While it was almost inexistent in young camels less than one year old, it increased with age and reached a maximum in 2 to 5 -year old camels. The authors showed that the infection has a significantly negative effect on PCV and on the overall status of the animals, confirming the pathogenicity of Trypanosoma evansi in dromedary camels. This irypanosome is almost the only species detected in the dromedary camel in Mali and it does not seem to cause infections in other animals reared in the same environment.
\end{abstract}

Key-words : Dromedary - Trypanosomosis - Trypanosoma evansi - Epidemiology - Pathological survey - Mali - Sahcl.

\section{BIBLIOGRAPHIE}

I. BANERJEE (S.), BJA'TIACHARJEE (R.C.), SINGH (T.I.). Hematological studies in the normal adult Indian camel (Camelus dromedarius). Am. $J$. Physiol. 1962, 203 : 1045-1047.

2. BOID (R.), EI AMIN (E.A.), MAHMOUD (M.M.), LUCKINS (A.G.) Trypanosoma evansi infections and antibodies in goats, sheep and camels in the Sudan. Trop. Anim. Hlth Prod., 1981, 13 : 141-145.

3. CAZALBOU (L.). Note sur un trypanosome du dromadaire au Soudan français. Bull. Acad. Med., 1903, 49:807-812.

4. Centre national d'élevage et de recherches vétérinaires, Nouakchott Rapport annuel, 1990.

5. Direction nationale de l'Elevage du Mali. Rapport annuel, 1988.

6. GATT-RUTTER (T. E.). Discases of camcls. II. Protozoal diseases. Vet Bull., $1967,37(9): 611-618$.

7. GODFREY (D.G.), KILLICK-KENDRICK (R.). Bovine trypanosomiasis in Nigeria. I. The inoculation of blood into rats as a method of survey in the Douga Valley, Benue Province. Ann. trop. Med. Parasit., 1961, 55 : 287-297.

8. GODFREY (D.G.), KILLICK-KENDRICK (R.). Trypanosoma evansi of camels in Nigeria : a high incidence demonstrated by the inoculation of blood into rats. Ann. trop. Med. Parasit., 1962, 56: 14-19.

9.GRUVEL (J.), BALIS (J.). La trypanosomiase à Trypanosoma evans chez le dromadaire au Tchad et ses principaux vecteurs. Revue Elev. Méd. vét. Pays trop., 1965, 18 (4) : 435-439.

10. MacLENANN (K.J.R.). A staining technique for identification of trypanosomes in thick blood films [Laboratory demonstration]. Trans. $R$ Soc trop. Med. Hyg., 1957, 51 : 301 .

11. MURRAY (M.), MURRAY (P.K.), McINTYRE (W.I.M.). An improved parasitological technique for the diagnosis of African trypanosomiasis Trans. R. Soc. trop. Med. Hyg., 1977, 71:325-326.

12. Service de l'Elevage et des Industries animales de la République Soudanaise. Rapport annuel, 1953.

13. WOO (P.T.K.). The haematocrit centrifuge technique for the diagnosis of African trypanosomiasis. Acta trop., 1970, 27 : 384-386.

DIALL (O.), BOCOUM (Z.), DIARRA (B.), SANOGO (Y.), COULIBALY (Z.), WAIGALO (Y.). Epidemiología de la tripanosomosis por Trypanosoma evansi cn el dromedario de Mali : resultados de encuestas parasitológicas y clínicas. Revue Élev. Méd. vét. Pays trop., 1993, 46 (3) : 455-461

Se estudió la epidemiología de la tripanosomosis camélida en las regiones de crianza de dromedarios en Mali. El estudio se basó en encuestas parasitológicas y clínicas, revelando tasas de infección globales de 9,5 p. $100(29 / 305)$ en el Sahel occidental (región I) y de 4,5 p. $100(28 / 627)$ en las zonas de Tombouctou y de Gao (región II). El porcentaje de hatos contaminados fue de 55 p. 100 en la región I y de 68 p. 100 en la región II. En algunos hatos, más del 50 p. 100 de los animales se encontraban infectados. Nuestras encuestas mostraron una tendencia al aumento de la prevalencia parasitológica con la edad : mientras que ésta es practicamente nula en los jóvenes de menos de un anõ, se observa un aumento paralelo a la edad, hasta alcanzar un máximo en la clase de 2-5 anõs. Los autores muestran que la infección tiene un efecto significativamente negativo sobre el hematocrito y el estado general, lo que confirma la patogenicidad del Trypanosoma evansi para el dromedario. Este tripanosoma fue el único (o casi el único) encontrado en el dromedario de Mali. No parece producir infecciones en otras especies presentes en el mismo medio.

Palabras claves : Dromedario - Tripanosomosis - Trypanosomosa evansi Epidemiología - Encuesta patológica - Mali - Sahel. 